\title{
Author Correction: Country-level social cost of carbon
}

\author{
Katharine Ricke (D), Laurent Drouet ID, Ken Caldeira and Massimo Tavoni \\ Correction to: Nature Climate Change https://doi.org/10.1038/s41558-018-0282-y, published online 24 September 2018.
}

In the version of this Article originally published, owing to a code error, the CSCC values for all income-dependent (that is, rich-poor) impact model specifications were incorrect, showing higher values relative to the preferred model rather than lower. These have now been recalculated for BHM SR RP, BHM LR RP and DJO RP, and Figs. 1, 2b and 3b, as well as Supplementary Figs 2, 4-6 and 9, have been updated to reflect these recalculations. In addition, the text reading, "The GSCC tends to be similar in both pooled and rich/poor specifications of the damages model, with the exception of SSP3, in which the estimated GSCC is much higher in the rich/poor specifications. The DJO specification of the economic impact function yields significantly higher GSCC values" has been modified to reflect this recalculation and now reads, "The GSCC is always lower using the rich/poor specifications of the damages model with confidence intervals that, in most cases, extend into the negative SCC range. The DJO specification of the economic impact function, which also estimates distinct effects for rich and poor countries, yields significantly lower GSCC values." The sentence beginning, "This results in higher (almost twice as much) global values of the SCC..." has also been modified to "In the case of the LR BHM specifications, this results in higher (almost twice as much) global values of the SCC..." The income thresholds and coefficients in the Supplementary Text, as well as the interpretation of the DJO sensitivity analysis results, have also been updated to reflect these changes. The authors thank Yixuan Zheng for identifying the code error.

\section{Additional information}

Supplementary information is available for this paper at https://doi.org/10.1038/s41558-019-0455-3.

Published online: 25 March 2019

https://doi.org/10.1038/s41558-019-0455-3

\section{Publisher Correction: Oscillating American winter temperatures}

Stephen Baxter

Correction to: Nature Climate Change https://doi.org/10.1038/s41558-019-0469-x, published online 22 April 2019.

In the version of this News \& Views originally published, the first author for ref. 3 was incorrectly indicated to be Baek-Min Kim, but should have been Mi-Kyung Sung. The DOI of the linked paper was also incorrectly stated as https://doi.org/10.1038/s41558-091-04615, but should have been https://doi.org/10.1038/s41558-019-0461-5. These errors have now been amended.

Published online: 1 May 2019

https://doi.org/10.1038/s41558-019-0492-y

\section{Publisher Correction: Strategies to reduce the global carbon footprint of plastics}

Jiajia Zheng (D) and Sangwon Suh (D)

Correction to: Nature Climate Change https://doi.org/10.1038/s41558-019-0459-z, published online 15 April 2019.

In the version of this Letter originally published, in Fig. 1 the label 'PPA 159 Mt' should have been 'PP\&A 159 Mt'. This has now been amended. 